\title{
Equality of opportunities for next generation researchers in Europe: a snapshot discussion motivated by the ERC statistics
}

\author{
Nedjeljka Žagar ${ }^{1, \mathrm{a}}$ and Thomas Schäfer ${ }^{2,3}$ \\ ${ }^{1}$ Meteorological Institute, CEN, Universität Hamburg, Grindelberg 7, 20144 Hamburg, Germany \\ ${ }^{2}$ Polymat, University of the Basque Country, Av. Tolosa 72, 20018 Donostia-San Sebastián, Spain \\ ${ }^{3}$ Ikerbasque, Basque Foundation for Science, 48013 Bilbao, Spain

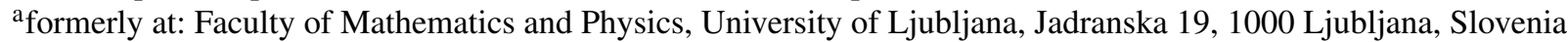

Correspondence: Nedjeljka Žagar (nedjeljka.zagar@uni-hamburg.de)

Received: 31 January 2020 - Revised: 4 May 2020 - Accepted: 29 May 2020 - Published: 17 June 2020

\begin{abstract}
This paper reflects on the results of a survey carried out within the COST TN1301 Sci-Generation network among the holders of ERC starting grants originating from countries of Central and Eastern Europe. The results of the survey reveal a greater personal satisfaction of the grant holders when carrying out the ERC-funded research in their native countries following successful $\mathrm{PhD}$ and/or post-doctoral periods in Western Europe and the USA. On the downside, a tenacious administration appears to be the largest burden for a new generation of successful researchers. It is discussed how the the statistics of ERC starting grants is a mirror of host countries' and host institutions' ability to attract and keep the most talented young researchers.
\end{abstract}

\section{Introduction}

During the previous decade, the European Cooperation in Science and Technology (COST, https://cost.eu, last access: 16 June 2020) funded several targeted networks targeting specific science policy strategies. One of the first targeted networks funded was TN1301 Sci-Generation (COST, 2013a) which was active during 2013-2017. Sci-Generation provided a platform for scientists at the beginning of their independent carriers to meet, discuss and act together with the aim of creating better career perspectives for the new generation of researchers in public research centres and universities, in the true sense of a European labour market, and particularly in countries with fewer opportunities. SciGeneration consisted of four working groups (WGs): WG1 focused on demographic issues, and in particular on issues that impede the career of young excellent researchers in the so-called COST "inclusiveness targeted countries" (COST, 2018); WG2 addressed career opportunities, i.e. career and funding perspectives for young researchers in the sense of a true European labour market; WG3 discussed issues related to research quality assessment, focusing on interdisciplinary research and its evaluation, but also in the wider sense on ethics both in evaluation and science; WG4 aimed at fostering synergy between European science policy platforms. Hence, TN1301 Sci-Generation attempted to cover the multiple facets and obstacles young researchers encounter when building their independent research careers within the actual European Research Area (ERA) (COST, 2013b). The proposal and the core of initial participants of TN1301 SciGeneration arose from a group of European Research Council (ERC) Starting Grant (StG) holders. Several members of the same group were also involved in founding the Young Academy of Europe (2020) meanwhile closely collaborating with Academia Europaea (2020). This paper is a snapshot of discussions within the WG1.

TN1301 Sci-Generation eventually counted on the participation of 31 of the 38 COST countries and as such was a unique platform to reflect on how young researchers achieve or struggle to achieve an independent research career. Of special importance for COST are the so-called "inclusiveness targeted countries (ITCs)". ITCs are countries that are on average less successful in attracting the EU funding and the list comprises $13 \mathrm{EU}$ member states which joined the EU in 2004 or later (European Union, 2020), the EU candidates Republic of Serbia, Montenegro, Turkey and Republic of North Macedonia, potential candidate Bosnia and Herzegovina (Euro- 
pean Commission, 2020a), as well as Portugal and Luxemburg.

An issue repeatedly addressed during the workshops and also discussions with national and European research agencies was the difference in the performance of north-western and south-eastern European countries to attract funding from the ERC. This is illustrated in Fig. 1 that compares the numbers of evaluated and approved applications for ERC StGs (European Research Council, 2020e) from the ITCs except Montenegro. The ratio between the two panels shows that an average success rate during the ERC StG calls was $6 \%-8 \%$ for Estonia, Portugal, Hungary, and Luxemburg; $3 \%-5 \%$ for Cyprus, Turkey, Poland, and Czech Republic; $1 \%-2 \%$ for Bulgaria, Slovakia, Romania, Serbia, Slovenia, and Croatia; and six countries (Bosnia and Herzegovina, Latvia, Lithuania, Malta, Montenego, Republic of North Macedonia) not hosting any ERC starting grant within the period reported. Considering only success rates hides another interesting aspect of these statistics, namely the number of applications evaluated from these countries. For example, from Portugal (success rate $5.8 \%$ ) $832 \mathrm{StG}$ proposals were evaluated between 2007-2019 of which 48 were funded; Luxemburg has a comparable success rate $(8.1 \%)$ but with only 37 StG proposals evaluated of which three were funded within the same period of time. Similarly, a success rate of $0 \%$ is very different in the case of Bosnia and Herzegovina, from where eight proposals were evaluated, compared to Lithuania, which in the period 2007-2019 submitted 52 proposals.

When comparing the success rate of the ITCs with the rest of Europe, the difference is not sharp. Iceland $(2.3 \%)$, Greece $(2.9 \%)$, Italy $(4.6 \%)$ remain below the $5 \%$ limit while Finland (5.7\%), Spain (7.1\%) and Norway (7.9\%) have success rates similar to the best performing ITCs. Yet, all other countries have success rates above $8 \%$, with Israel and Switzerland systematically performing with over $20 \%$ success rate. Also in the rest of Europe similar success rates may hide significant differences in number of applications. For example, $14.5 \%$ of the 956 proposals evaluated from Austria were successful. The United Kingdom has a similar success rate $(12.6 \%)$ but with more than seven times more applications evaluated (7053). On the other hand, Italy and Germany got a similar number of proposals evaluated between 2007-2019 (5359 and 5552, respectively) but with very differing success ( $4.6 \%$ and $12.8 \%$, respectively). In general, it can be observed that the number of evaluated applications from ITCs is one to two orders of magnitude lower than that of the rest of Europe.

The ERC grant statistics has been presented at many occasions; we provide here the links to several presentations (European Research Council, 2020a, b, c, d). All data is publicly available (European Research Council, 2020f). An interesting feature of the statistics is the fact that the ITC success rate has not changed significantly since the start of the ERC program. In particular, it has not changed since 2013 when a survey among the ERC StG holders from countries of Eastern and South-Eastern Europe, to be discussed here, was carried out. The survey was first presented at the 25th anniversary conference of Academia Europaea in September 2013 in Wroclaw at the session entitled "Young academy, future visions", within the collaboration between the Young Academy of Europe and Academia Europaea. The goal of the survey was to identify factors influencing the grantees' choice of host country and host institution, and their general satisfaction with the research environment in which they realized their ERC grants.

In what follows, we discuss the results of the survey and the associated discussions within WG1 of Sci-Generation. The goal is to contribute our views and perspective to the special issue of diversity and equality in the geosciences in time (spring 2020) when equality, diversity and inclusion in European research area are as relevant as ever.

\section{Survey among the ERC StG holders in Inclusiveness Targeted Countries (ITCs)}

There is an ongoing discussion regarding the (under) performance of a country in the ERC competition and it is difficult, if not impossible, to strictly relate success rates of StG proposals from different countries with the quality of research and/or research infrastructure. Yet, as was outlined above, the success rate of ITCs tends to be lower than in the rest of Europe. This note discusses some factors influencing the success rate based on a survey among a relatively small number of colleagues and personal experiences, but benefitting from the discussions held within Sci-Generation. We do not aim at applying scientific methodology here, neither was any scientific method applied to prepare the questionnaire of the survey. The small number of participants and the open responses to the questions do not provide a rigorous basis to analyze the results statistically.

Similarly, no attempt has been made either to relate the number of StG proposals evaluated to the number of registered researchers in the various countries, their gross national income, investment in research and/or population. Such analysis goes beyond the scope of this paper and is performed by European Commission (for example, European Commission, 2020b). This note is rather a qualitative summary of the survey and an overview of discussions among ERC-grantees involved in Working Group 1 of the TN1301 Sci-Generation. Rather than comparing numbers, we discuss a few factors identified by ERC grantees to be important for their working environment and success in their native countries of Central and East Europe.

The survey was carried out in September 2013 and information about the grants and names of the grantees was collected at the time from the ERC webpage. About 30 grant holders were contacted and 23 responded to the survey. Appendix A lists the seven questions that were asked. They were formulated based on the first author's own experience and 
(a) Numbers of submitted ERC starting grants in the so-called COST

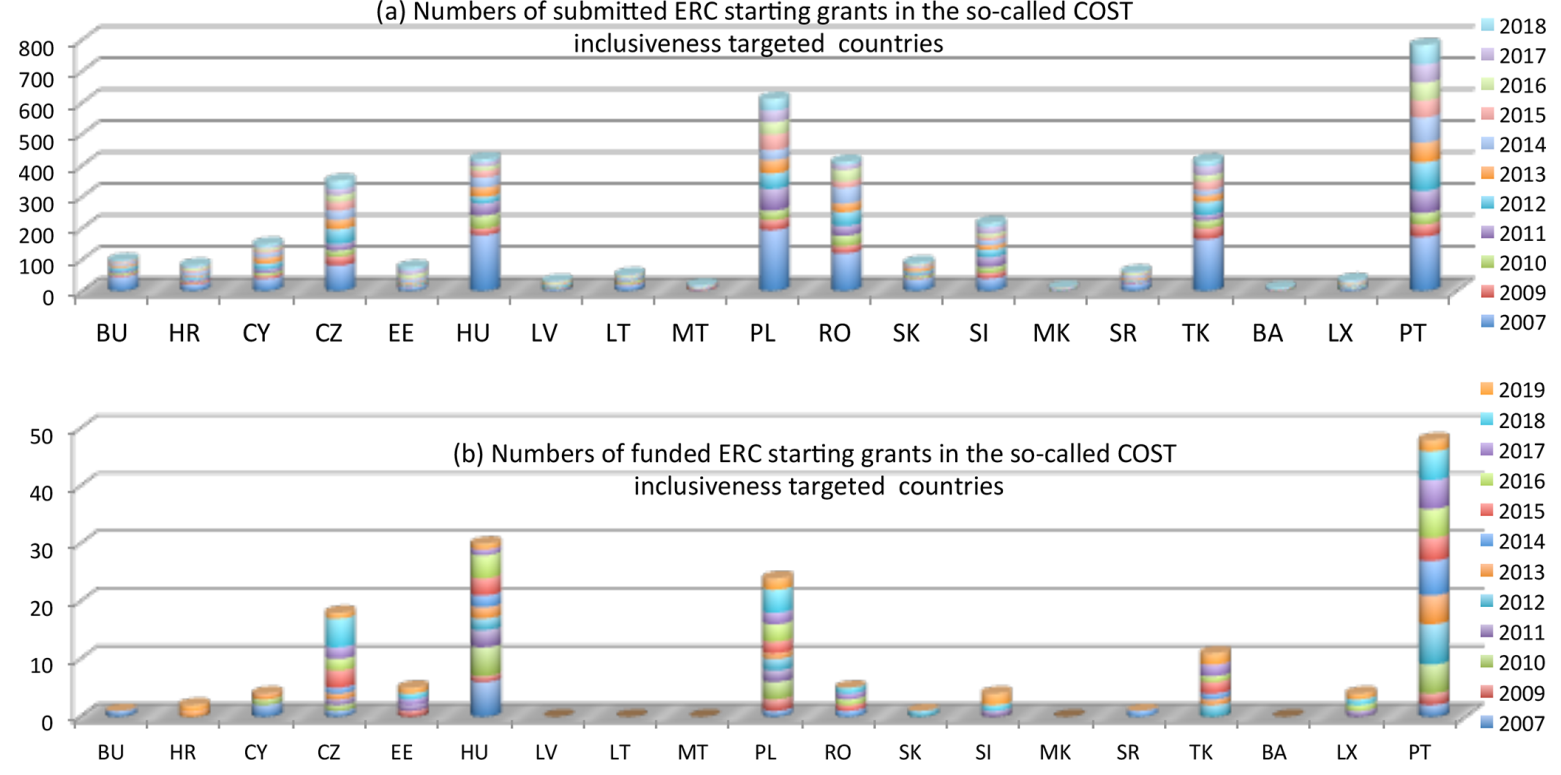

Figure 1. Numbers of submitted (a) and funded (b) ERC starting grants in the so-called COST inclusiveness targeted countries.

judgment on what were important factors for an early-stage independent academic career. This may seem too subjective. However, we would like to point out that being embedded in a unique network like TN1301 Sci-Generation in which discussions were highly interdisciplinary and with participants from a large variety of cultural and national backgrounds, this questionnaire tacitly addressed which was understood as possible key aspects to be addressed. The questions included:

- Educational record and work experience of the ERC grant holder (principal investigator, PI)

- Relation to the host country (HC)

- Satisfaction with the working conditions and life in the $\mathrm{HC}$ in general

- Relation to the research world outside the HC, especially to neighbouring countries

A paper with a discussion of the results of the survey was published in early 2015 on the webpage of Sci-Generation that is no longer available. Here we present an updated version of that paper.

\section{Discussion of the response to the survey among the ERC StG holders in ITCs}

\subsection{Personal background of PIs and relation to the host country (HC)}

All but one of the PIs interviewed had returned to their HC after spending an extended period abroad (in a non-ITC), either doing their $\mathrm{PhD}$ or post-doc, or both. All but one PI, who is an author of this paper, were conducting their ERC-funded research in his/her native country. Asked to enumerate and rate reasons for choosing their $\mathrm{HC}$ (host institution, quality of working conditions, personal reasons such as family), all PIs chose "personal reasons" as the main factor influencing their choice, although sufficient infrastructure or the research topic (in humanities) were also playing a significant role. As one of the PIs replied: "I feel that by staying in my home country and being active, I am making a difference." Words used to describe one's relationship to the HC are "optimism, empowerment, trust, education, insurance, active citizenship".

This reveals that a strong motivation for the choosing the $\mathrm{HC}$ is the impact a PI expects to have with her/his research in their immediate environment. This is not necessarily unique for the countries encompassed by this survey. It is probably an honest thing to admit that a majority of scientists have their personal life rather high on the priority list when they are choosing the $\mathrm{HC}$. Unless working conditions are objectively really poor, being close to one's family and friends is important in the period when many people start their families. What is somewhat different for the countries in focus is a special point in history of these countries that have seen great changes in terms of improvement of research infrastructure, changes in scientific policy and large shifts in societal values and rules since their entrance into the EU.

This impact is considered to be significant irrespective of the field of research due to the dynamics the HC is undergoing. Compared to fellow researchers in non-ITCs which socially and economically have been moving with a more stable pace, one might conclude that the motivation of PIs working in ITCs is enhanced as "making a difference" in their HC or $\mathrm{HI}$ is a motivation on top of the one any scientist aspires 
to with regard to the purely scientific impact of their work. Therefore, one might consider PIs in ITCs as particularly motivated and as such possibly also particularly willing to cope with obstacles which their fellow PIs in non ITCs face to a much lesser extent. One would thus expect that the pool of PIs with such attitude would be considered a unique opportunity to foster the transition, as far as research is concerned, from an ITC to a non ITC.

A majority of PIs thinks that he/she could fulfill the research goals of their grant better at another HI and in another HC. This is in line with the assumption that other factors (making an impact, work-life balance) are likely to outweigh absolute scientific productivity. Hardly any of the PIs has contacts with scientists from their field or related fields in neighbouring countries in the region but keep contacts with their former mentors and collaborators in Western Europe and the USA. The latter might be representative for the actual situation of the ERA in which, after all, science is still done widely within national boundaries although the aim is a European research landscape.

\subsection{Working conditions in the host country (HC) and host institution (HI)}

The four principal aspects determining working conditions of the PIs and their groups were reported to be research infrastructure; difficulties in hiring qualified staff; administrative burden; and work-life balance. Among these four aspects, dealing with administrative issues at the $\mathrm{HI}$ and other institutions of the $\mathrm{HC}$ was pinpointed as the biggest challenge. The second biggest problem identified was hiring group members to form new research teams from scratch in small countries with rapidly developing research infrastructure enabled by the European structural funds. Overall, the research infrastructure and work-life balance was generally considered as being good.

Taking into account that the PIs interviewed had previous experience from non ITCs, such as western/northern EU countries and USA, they apparently experienced more bureaucracy in ICTs, or in other words, administrative hurdles seem to be one of the clear weak-points of ITCs. When administrative support is generally weak it renders the administrative burden the most serious disadvantage. Weak administrative support is to some point understandable where contact with European funding schemes has been extremely limited. Some PIs have in fact noticed that the administration also develops along the way with the ERC grant scheme and general development of research systems in their native countries. Indeed, the COST targeting network BESTPRAC (https://bestprac.eu/home/, last access: 16 June 2020) was active in recent years and provided the training and exchange of best practices for administrative staff in support of excellent research.

However, the situation may turn into an ouroboros if administrative support is expected to progress on the basis of "learning by doing": an administration that cannot live up to the needs of excellence science will not be supportive, rendering excellence science less probable which in turn decreases exposure of the administration to European funding schemes.

We therefore suggest that this vicious circle can only be broken by actively training the administration on a lo$\mathrm{cal} /$ regional/national scale in order to create in this way an infrastructure that addresses the needs of excellent science both in short term needs as well as a long-term vision. Here, the impact of active researchers, not necessarily only those that are PIs of ERC-funded research, would obviously be highly valuable as they are the most competent to represent the needs of excellent science, a circumstance that does not only apply to ITCs. One of the outcomes of TN1301 SciGeneration was that in this respect the conditions across Europe are highly heterogenous and we are far from what could be a truly prosperous ERA. While in this process the EC can take, and actually takes, supportive actions, the responsibility to streamline administration lies in the hands of the local and regional institutions, embedded in a national vision.

Problems with hiring qualified international post-docs are primarily associated with the intrinsic problem of salaries being lower than, for example, in western Europe, as well as a lack of tradition and reputation of the HIs, making them less attractive compared to renowned HIs in the west. In this case a clear difference seems to exist between ERC grantees that are consolidating their independent research career by forming their own group, and young researchers that are still accumulating post-doctoral experience before seeking a position as senior scientist. Among various countries, PIs from Poland have expressed greatest satisfaction with their working conditions, salary levels and hiring possibilities, all in comparison with neighbouring countries.

\section{Discussion of challenges for the new generation of European researchers}

Obstacles faced by a new generation of European research leaders are shared across Europe. Challenges were summarized by WG1 of Sci-Generation as follows:

\section{Lack of resources}

2. Lack of measures to stimulate excellence amongst next generation researchers

3. Lack of excellent research environment and training of the next generation researchers

\section{Lack of independence for next generation researchers}

In ITCs the gross domestic expenditure on R\&D is well under the European goal of $3 \%$. But the "lack of resources" goes beyond the funding: a lack of trained research administration to support researchers during their grant preparation 
and during project implementation can force project leaders to turn into part-time administrators and lose in this way time and energy that would be better employed in doing science. A lack of having ready access to the experience of fellow researchers that attracted funding for excellence science is another parameter that can prove decisive even at the moment of considering whether to write a proposal at all.

The problem of stimulating excellence amongst next generation researchers is a relatively recent topic in most ITC countries (COST Targeted Network TN1301, 2015). A fundamental condition for stimulating excellence is certainly transparency in any of the aspects relevant to a scientific career:

- Transparency during the evaluation of research grant applications ensures an unbiased evaluation process. However, scientists in ITC countries are more often obliged to write their grant applications in their national language, limiting in this way significantly the pool of possible independent reviewers.

- Hiring processes for permanent or senior research positions are less often internationally advertised and often lack transparency. They may in this way suffer from academic inbreeding, such that new scientific leaders are not necessarily hired on the basis of their scientific merit. Even worse, such senior scientists are less likely to prove good mentors for the next generation researchers, and the lack of best practices is perpetuated.

An obvious solution would be to reform the existing research environment and establish best practices that foster earlystage independence of young researchers. However, reforms, when made, may be led by a scientific establishment with no real interest in changing the status quo, and thus most probably lead nowhere. In such hierarchical environments, where early-career investigators do not have enough independence to develop independent research lines, writing a strong, internationally competitive ERC StG proposal is a comparatively far more demanding endeavour. Furthermore, a strong role of the local scientific networks may hinder international mobility as people might loose their existing position and related privileges when taking a leave for a temporary position abroad.

The situation may be further aggravated by national research strategies pursuing arbitrarily set (numerical) targets, the infamous "indicators", and a short-term research policy and funding: a temporary lack of resources quickly leads to discontinuity and incoherence of national research programs, which in turn frustrates attempts to establish lasting excellence and motivation amongst the next generation researchers. The lack of priorities for allocating funds to support excellent research at national levels is preventing the recognition and self-awareness in young researchers from these countries. Their low visibility, and the low visibility of their home countries themselves can contribute to bias when reviewing research proposals of researchers from ICs and in this way lower their success rates. Furthermore, a lack of incoming excellent senior researchers ("excellence attracts excellence") contributes to an insufficient mentoring of young scientists in preparing European grant applications.

It must be stressed that the issues raised during this survey amongst PIs in ITCs do also exist in many non ITCs. It was discussed in Introduction that there was no sharp distinction between ITCs and other European countries when it comes to success rates during ERC StG calls. Similarly, the issues raised in this survey can possibly be found anywhere across Europe and may even differ within countries. This highlights once more the need for supporting excellence science also in a bottom-up approach rather than expecting that programmes like the ERC-scheme will be the sole measure to disrupt incrusted and outdated research environments where they exist.

\section{Summary}

Competition on the research job market as known in countries of Western and Northern Europe is less developed in countries of Central and Eastern Europe and we can hardly talk about a truly European Research Area in these countries. Here, language is only a minor barrier; opened positions in academia may be announced only formally and a circle of people creating scientific policy may still be dominated by individuals and groups that emerged from non-democratic systems before 1990 .

A great impact of the ERC starting grants in these countries is an empowerment of ERC grant holders. In particular, possibility to change the HI, even if often not feasible, is a great means for giving a voice to researchers at the start of an independent carrier. In this respect, an ERC StG is like an open window for change. However, as most of the countries are small and a choice of potential HIs is limited, ERC starting grant holders need to be adequately embedded in local systems and supported by their senior colleagues and former mentors in order to receive recognition.

Although many changes have occurred across Europe since 1990, administration at many places still play a role of a controller rather than a supporter to scientists. ERC grants are formulated with an idea to minimize the amount of the required administrative work; the amount of real administration is controlled by local rules and the role of local project administrator cannot be underestimated. This applies not only to the COST inclusiveness targeted countries, and it was addressed by COST through a targeting network BESTPRAC that during 2014-2019 worked on improvements of European research administration.

By definition, the ERC grant system promotes great ideas and excellent research proposals from researchers from around the world, without directly interfering with the HIs or HCs. The grant holders are supposed to choose the HI and $\mathrm{HC}$ that best support the realization of their project goals. In 
reality, a strong motivation for choosing the $\mathrm{HC}$ among ITCs is the return to the native country where the work-life balance and the impact of a PI in the society may be maximized. ERC grants can, hence, be considered as a mirror to HIs and HCs as regards their ability to keep and to attract back their most talented young researchers.

According to the factors listed above, one can expect that Eastern European countries remain non-attractive HCs to other than their nationals for some years to come. This may be frustrating where constructive efforts are actually being made to rise the HC's attractiveness. However, a science policy that does not expect short-term benefits in a process that naturally takes time and that will rigorously pursue the quest for excellence and adequate working conditions for next generation researchers, will eventually be fruitful and pay off. Changing attitude of science policy elites towards research excellence and excellent researchers may be a great, nonscientific result of the ERC scheme in, and possibly not only, COST inclusiveness targeted countries. 


\section{Appendix A}

Questions asked the ERC Starting grant holders in several countries of east and south Europe (September 2013)

1. Are you located in your home country (HC)? If so, did you return from abroad i.e. spent time abroad $(\mathrm{PhD}$ studies, post-docs) prior to ERC StG?

2. If located in your home country, what are the reasons for it.

a. optimal research capacities to realize my grant (the same or better than elsewhere),

b. general quality of working conditions

c. personal reasons (such as closiness to family, life quality due to family support).

If several reasons apply, can you grade them. Did you consider also other HCs when applying for ERC StG?

3. Do you sometimes (if so, how often) regret the choice of $\mathrm{HC}$ and host institution (HI)?

4. Problems (if any) which you face at work are mostly related to: a. infrastructure conditions for carrying out your research,

b. difficulties in hiring qualified $\mathrm{PhD}$ students and post-docs,

c. administrative work in handling your HI administration,

d. simply managing the life (Is your salary strictly defined by the national laws and un-effected by grant funding?)

5. Do you think that you could accomplish your ERC project goals better if you were in another $\mathrm{HC}$ or $\mathrm{HI}$ ?

6. How do you compare working condition in your HC with those in the neighbouring countries? Do you have a collaboration or you care to collaborate with neighbouring countries and contribute to regional science collaboration?

7. Are you a member of YAE? If not, why not? What do you think YAE can do to support ERC StG of "New Europe"? Please discuss where possible. 
Data availability. Data used in Fig. 1 are available from http://erc. europa.eu/projects-and-results/statistics (European Research Council, 2020f). Results of the survey are available on request from the authors.

Author contributions. NZ conceived the original idea of the present research, analysed and validated the data, elaborated the interpretations of the results. NZ and TS contributed in writing and reviewing the manuscript.

Competing interests. The authors declare that they have no conflict of interest.

Special issue statement. This article is part of the special issue "Diversity and equality in the geosciences (EGU2019 EOS6.1 \& US4, AGU2018 ED41B, JpGU2019 U-02)". It is a result of the EGU General Assembly 2019, Vienna, Austria, 7-12 April 2019.

Acknowledgements. The authors would like to thank all colleagues who as ERC grantees responded to the questionnaire, all those that actively participated in the COST Targeted Network 1301 "SciGeneration", and COST for constructive discussions. We also thank an anonymous reviewer and Liviu Matenco for their comments on the manuscript and to the topical editor Claudia Jesus-Rydin for her support.

Financial support. This research has been supported by the COST Targeted Network 1301 "Sci-Generation".

Review statement. This paper was edited by Claudia Jesus-Rydin and reviewed by two anonymous referees.

\section{References}

Academia Europaea: https://www.ae-info.org, last access: 15 May 2020.

COST: TN1301-Next Generation of Young Scientists: towards a contemporary spirit of R\&I (Sci-GENERATION), available at: https://www.cost.eu/actions/TN1301 (last access: 15 May 2020), 2013a.

COST: Memorandum of Understanding for the implementation of a European Targeted Network designated as COST Action TN1301: Next Generation of Young Scientist: towards a contemporary spirit of R\&I (Sci-GENERATION), available at: https://e-services.cost.eu/files/domain_files/TN/Action_ TN1301/mou/TN1301-e.pdf (last access: 15 May 2020), 2013b.
COST: Inclusiveness Target Countries (ITC) Conference Grants User guide, available at: https://www.cost.eu/wpcontent/uploads/2018/11/Inclusiveness-Target-Countries-

Conference-Grants-userguide-V2-003-Oct-2018-1.pdf (last access: 15 May 2020), 2018.

COST Targeted Network TN1301: Paths to independence of young European researchers and research teams: mapping of the current situation, Sci-Generation, Working Group 1, Progress report published on 25 March 2015, available from COST or at: https://drive.google.com/file/d/ 1DUITSjwLxDnNsZ71I1R-crn-vmCGLR9R/view?usp=sharing (last access: 16 June 2020), 2015.

European Commission: Enlargement, available at: https: //ec.europa.eu/environment/enlarg/candidates.htm, last access: 15 May 2020a.

European Commission: From HORIZON 2020 to HORIZON EUROPE. Monitoring Flash, available at: https: //ec.europa.eu/research/evaluations/pdf/archive/h2020_ monitoring_reports/h2020_monitoring_flash_092018.pdf, last access: 15 May 2020b.

European Research Council: "What is the ERC?", available at: https://erc.europa.eu/sites/default/files/document/file/ Equality_of_opportunity_in_European_Research_Council_ Competitions_Barbara_ROMANOWICZ.pdf, last access: 15 May 2020a.

European Research Council: ERC Funding Opportunities: Supporting excellent researchers all over Europe. The ERC's quest to support excellent researchers all over Europe, available at: https://erc.europa.eu/sites/default/files/content/pages/pdf/1_ Budapest_Opening_Eva_Kondorosi.pdf, last access: 15 May 2020b.

European Research Council: ERC Funding Opportunities: Supporting excellent researchers all over Europe. PANEL DISCUSSION: Perspectives on widening European participation in ERC competitions. Supporting Evidence, available at: https://erc.europa.eu/sites/default/files/content/5.ERC_event_ Tallinn_Mart_Saarma.pdf, last access: 15 May 2020c.

European Research Council: ERC - Supporting 8 years of Excellence in Frontier Research and the Czech Republic, available at: https://www.google.com/url?sa=t\&rct=j\&q=\&esrc=s\&source= web\&cd=8\&ved=2ahUKEwjttIuNm9noAhWMMewKHY30A WUQFjAHegQICBAB\&url=https://www.h2020.cz/storage/ download/f616ea4bb84341a541992b6eb5720842254734bf\& usg=AOvVaw0LFPMJebucbBGu9Le0QbM0, last access: 15 May 2020d.

European Research Council: ERC Funded projects, available at: https://erc.europa.eu/projects-figures/erc-funded-projects, last access: 15 May 2020e.

European Research Council: Statistics, available at: http://erc. europa.eu/projects-and-results/statistics, last access: 15 May $2020 \mathrm{f}$.

European Union: About the EU, available at: https://europa.eu/ european-union/about-eu/, last access: 15 May 2020.

Young Academy of Europe: http://www.yacadeuro.org, last access: 15 May 2020. 